> Les cellules microgliales sont les macrophages résidents du système nerveux central (SNC). La microglie a une fonction cruciale dans l'homéostasie du SNC adulte, mais des études récentes montrent que les cellules microgliales pourraient aussi jouer un rôle dans le développement du SNC. Dans cette revue, nous décrivons comment les précurseurs microgliaux ont été découverts, comment ces cellules envahissent le système nerveux central chez l'embryon, quelles sont leurs interactions cellulaires précoces et quelles sont leurs fonctions qui ont été identifiées chez l'embryon. Ces découvertes ouvrent une nouvelle voie de recherche dans l'analyse des mécanismes qui régulent le développement embryonnaire du SNC. <

Les cellules microgliales sont les macrophages résidents du système nerveux central (SNC). Elles représentent environ $10 \%$ des cellules gliales du cerveau. Depuis leur mise en évidence par Pio del Rio-Hortega (18821945), de nombreux travaux ont démontré leur fonction macrophagique dans le tissu nerveux [1]. En situation physiologique, les cellules de la microglie interagissent continuellement avec leur environnement en projetant des extensions qui «analysent » les cellules environnantes [21] $(\rightarrow)$.

$(\rightarrow)$ Voir la Synthèse de $\varepsilon$. Audinat et l. Arnoux, page 153 de ce numéro

Ce comportement de surveillance leur permet de détecter toute altération de leur environnement immédiat. $\varepsilon$ n réponse à une situation pathologique, ces cellules vont rapidement proliférer et s'activer pour adopter un phénotype analogue à celui des macrophages activés. Elles sont alors capables d'éliminer les débris cellulaires (fonction macrophagique), et de sécréter des facteurs immunorégulateurs (cytokines) et des facteurs de croissance. Cependant, si leur activation persiste, elles peuvent aussi avoir un rôle néfaste pour les autres cellules du SNC en sécrétant des cytokines pro-inflammatoires et des facteurs cytotoxiques [1].

La présence de ces cellules dans le SNC embryonnaire est connue depuis les années 1930, mais leur processus d'in-

\section{Cellules microgliales et développement du système nerveux central chez l'embryon}

Pascal Legendre ${ }^{1,2,3}$, Hervé Le Corronc ${ }^{1,2,3,4}$

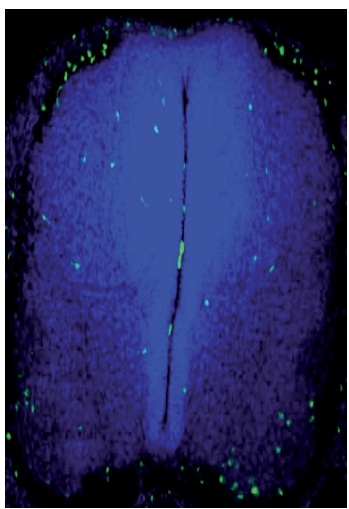

${ }^{1}$ Inserm UMR-S 1130, université Pierre et Marie Curie (UPMC), 9 , quai Saint-Bernard, Paris, France ; ${ }^{2}$ CNRS, UMR 8246, UPMC, 9, quai Saint-Bernard, Paris, France ; ${ }^{3}$ UPMC UM CR18, 9, quai SaintBernard, Paris, France ; ${ }^{4}$ Université d'Angers, 40, rue de Rennes, Angers, France.

pascal.legendre@snv.jussieu.fr

vasion et leur rôle dans le développement précoce du SNC commencent seulement à être élucidés. Un nombre croissant de données indiquent, notamment, que la microglie embryonnaire pourrait jouer un rôle important dans le développement normal des réseaux de neurones [2] et, peut-être, représenter un des acteurs impliqués dans des pathologies psychiatriques comme la schizophrénie et l'autisme [2, 3].

\section{Origine extra-embryonnaire de la microglie}

Ce n'est que récemment que l'origine hématopoïétique de la microglie a été démontrée $[4,5]$. On savait depuis les travaux de Pio del Rio-Hortega que ces cellules envahissent le SNC de l'embryon chez l'homme, la souris et le poulet à des stades précoces du développement, bien avant l'hématopoï̀se définitive $[1,6]$. Ces observations posaient la question de l'origine des cellules microgliales chez l'adulte [1]. Provenaient-elles de l'hématopoïèse primitive et/ou de l'hématopoïèse définitive (Figure I) ? C'est en démontrant que les précurseurs de la microglie avaient une origine extra-embryonnaire (le sac vitellin, SV) que Ghinoux et al. [7] ont suggéré que la microglie provenait de l'hématopoïèse primitive. Ces auteurs ont montré que les cellules microgliales ne provenaient pas de précurseurs de la moelle osseuse, en irradiant de manière sub-létale des nouveau-nés et en greffant à ces animaux la moelle de souris congéniques (souris génétiquement identiques ne différant que par l'expression d'un marqueur génétique). Ils ont ensuite démontré que ces cellules proviennent principalement du sac vitellin, et non pas du foie fœtal, en utilisant la technique dite du fate mapping (ou suivi du destin cellulaire), qui consiste à exprimer 


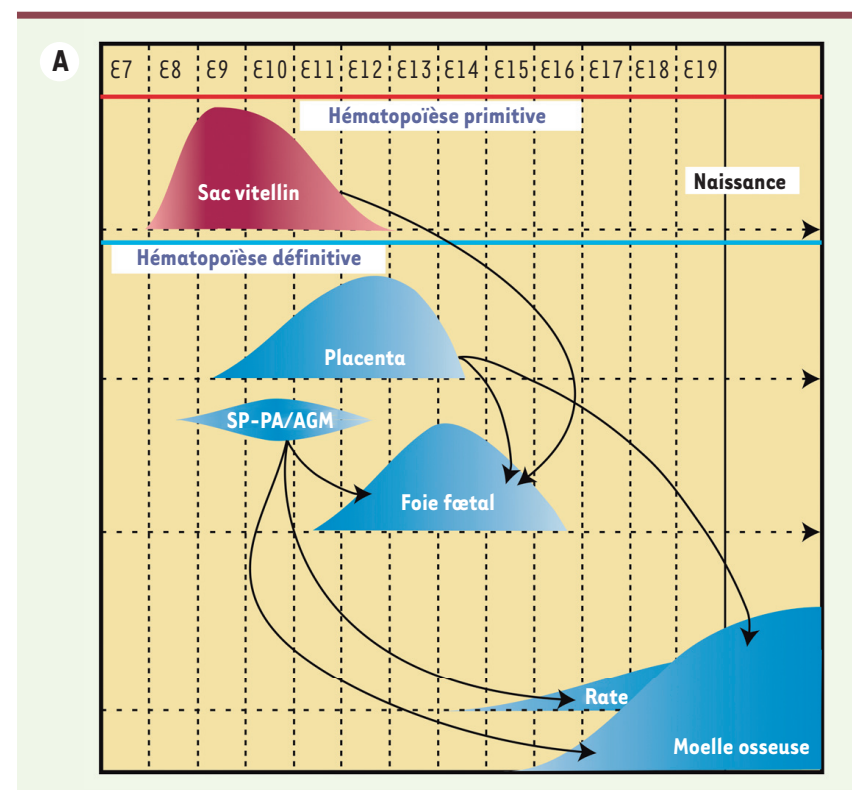

B

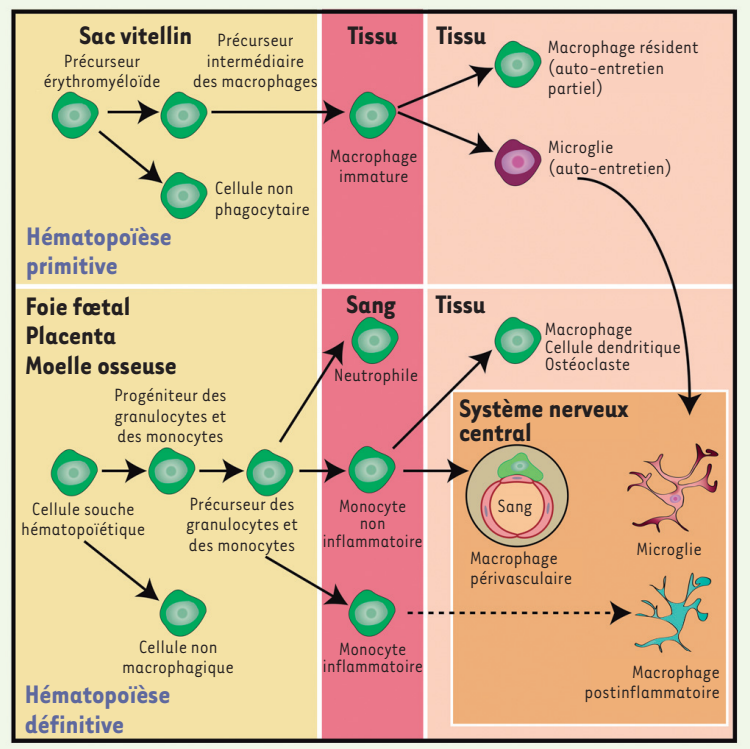

Figure 1. Développement hématopoïétique, origine extra-embryonnaire de la microglie et lignage macrophagique. A. Synopsis de l'hématopoïèse au cours du développement chez l'embryon de souris. Aux $7^{e}-8^{e}$ jours de gestation $(\varepsilon 7-\varepsilon 8)$, le sac vitellin, annexe extra-embryonnaire, est capable de produire les premières cellules hématopoḯtiques. Cette hématopoïèse précoce du sac vitellin est appelée hématopoièse primitive, par opposition à celle dite définitive, qui dérive des cellules souches multipotentes produite par le placenta et la région intra-embryonnaire, appelée région de la splanchnopleure para-aortique/aorte-gonades-mésonéphros (SP-PA/AGM). Ces cellules migrent ultérieurement dans le foie fœtal et la moelle osseuse pour y produire toutes les cellules hématopoḯtiques. C'est le foie fœtal qui constitue le site principal de l'hématopoièse à partir du stade $\varepsilon 10$, mais la moelle osseuse sera le site exclusif de l'hématopoïèse à la naissance et pour toute la vie. B. Les cellules microgliales (pourpre) sont dérivées des phagocytes immatures (section sac vitellin ; vert) provenant de l'hématopoïèse primitive au niveau du sac vitellin chez l'embryon. Ces phagocytes immatures migrent le long du système vasculaire primitif dans le neuro-épithélium. Leurs progéniteurs sont des précurseurs érythromyéloïdes qui n'existent que chez l'embryon et dont les potentialités de différenciation se limitent aux lignées macrophagique et érythrocytaire. À la différence des précurseurs érythromyéloïdes, les cellules souches multipotentes de l'hématopoïèse définitive sont localisées dans la moelle osseuse (section foie fœtal, placenta, moelle osseuse ; vert). Chez l'adulte, la majorité des macrophages, incluant les macrophages périvasculaires du cerveau (section système nerveux central ; vert pâle), qui sont confinés par la barrière hémato-encéphalique dans un espace entourant les vaisseaux sanguins, dérivent de l’hématopoï̀se définitive. Dans des conditions normales, la population microgliale adulte est une population cellulaire qui s'auto-entretient et il n'y a pas d'apport extérieur provenant du réseau vasculaire. Mais, dans des conditions pathologiques, comme dans les maladies auto-immunes ou en réponse à une perte massive de microglie, des monocytes CRR2+ inflammatoires provenant de l'hématopoïèse définitive peuvent entrer dans le système nerveux central. Ces monocytes deviennent des macrophages post-inflammatoires (en bleu) et acquièrent des caractéristiques proches de celles de la microglie (d'après [20]).

spécifiquement un marqueur dans les cellules dérivant du SV au moyen du système Crelox (pour plus de détails, consulter [6]).

L'origine extra-embryonnaire de la microglie a été confirmée par Kierdorf et al. [8]. S'appuyant sur un ensemble sophistiqué de souris transgéniques, ces auteurs ont pu montrer que les cellules progénitrices de la microglie correspondaient à des précurseurs érythromyéloïdes présents dans le sac vitellin dès le $8^{e}$ jour de gestation (Figure 1). Ils ont, par ailleurs, identifié deux facteurs de transcription, PU.1 et IRF8 (interferon regulatory factor 8), qui se sont révélés indispensables pour le développement des cellules microgliales, IRF8 agissant en amont de PU.1. La maturation des précurseurs érythromyéloïdes se déroule en deux temps. D'abord, ces précurseurs donnent naissance à des cellules immatures CD45+ et c-kit ${ }^{\text {lo }}$ (cellule Al). Puis, les cellules Al vont se différencier en cellules $A 2$ caractérisées par une répression complète de l'expression de c-kit et l'acquisition de l'expression de $\mathrm{CX}_{3} \mathrm{CR} 1$, le récepteur de la fractalkine $\left(\mathrm{CX}_{3} \mathrm{CLl}\right)$. La fractalkine est une cytokine de type chimiokine. Les chimiokines ont une fonction d'attraction (chimiotactisme) et contrôlent l'état d'activation des cellules du système immunitaire. Pendant le développement postnatal, le couple $\mathrm{CX}_{3} \mathrm{CRl} /$ $\mathrm{CX}_{3} \mathrm{CLl}$ joue un rôle important dans les interactions microglie/neurones et semble réguler le rôle de la microglie dans la neurogenèse, la synaptogenèse et l'axogenèse (voir pour revue [9]). Les cellules de type A2 prolifèrent et se différencient en précurseurs macrophagiques au sein du sac vitellin. Ces précurseurs macrophagiques vont donner des macrophages immatures, puis devenir des cellules microgliales en envahissant le SNC embryonnaire. Pour envahir le SNC, ces cellules utilisent des métalloprotéinases spécifiques de la matrice extracellulaire. 

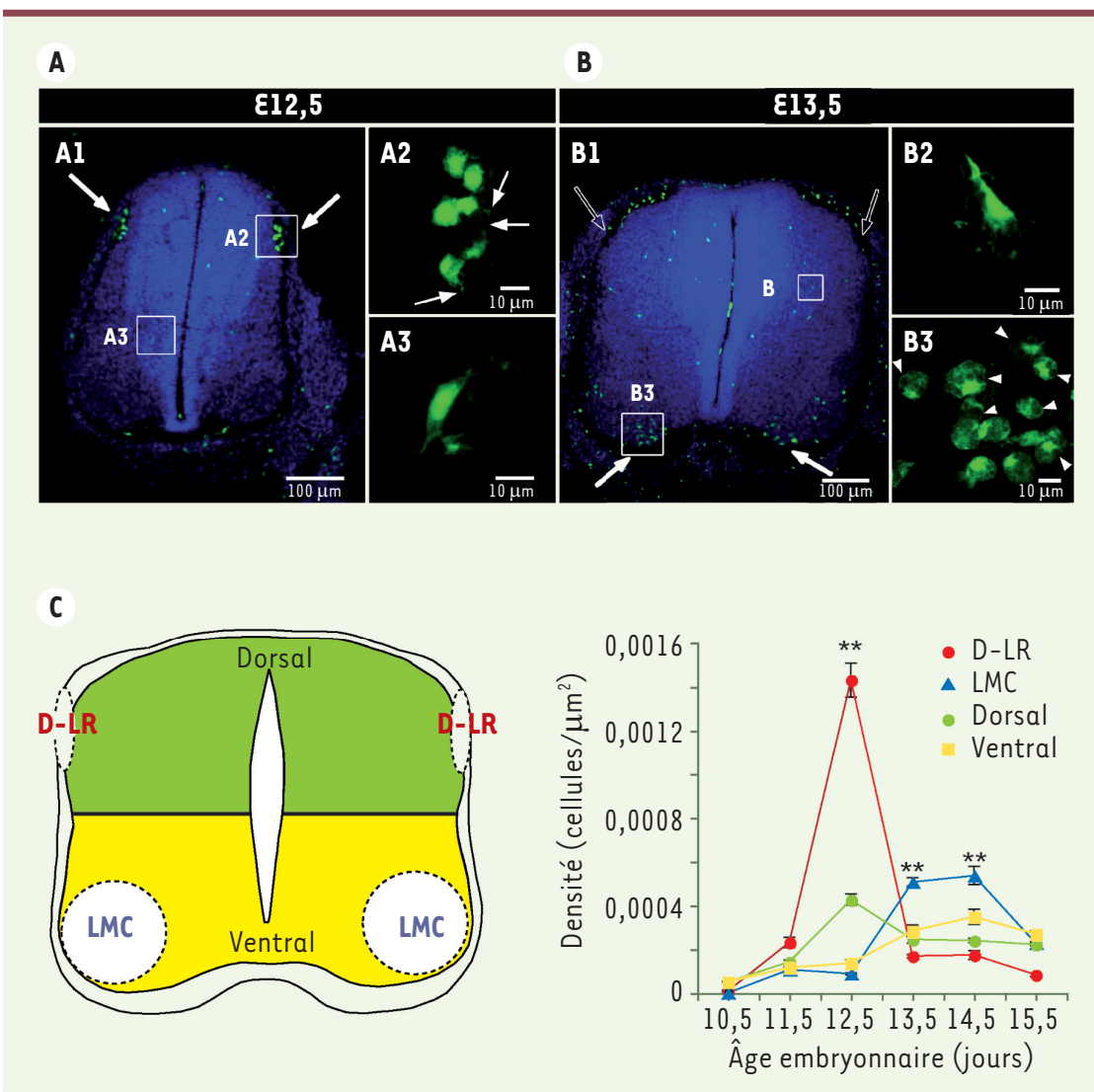

Figure 2. Patron temporel d'invasion de la moelle épinière embryonnaire par les cellules microgliales. A-B. Coupes de moelle épinière d'embryons de souris exprimant la protéine fluorescente eGFP à 12,5 jours ( $A$ ) ou à 13,5 jours (B) de gestation. On notera que les cellules microgliales sont peu ramifiées à ces stades (A2, A3, B2). À $\varepsilon 13,5$, les cellules localisées dans la colonne motrice latérale (B3) ont une forme amiboïde et contiennent de nombreuses vacuoles. Pour montrer la morphologie de la moelle épinière, les noyaux ont été visualisés par un marquage au DAPI (4',6'-diamidino-2-phénylindole) (bleu). C. Schéma à gauche: une coupe de moelle épinière décrivant les zones de quantification de l'évolution de la densité des cellules microgliales avec l'âge (D-LR: région dorso-latérale; LMC : colonne motrice latérale). Graphique de droite: quantification de l'évolution de la densité des cellules microgliales en fonction de l'âge de l'embryon. On notera une augmentation transitoire de la densité microgliale dans la région dorso-latérale et la colonne motrice latérale (d'après [10]).

\section{Les cellules microgliales envahissent le SNC à des stades précoces du développement embryonnaire}

Il a fallu attendre la fin des années 2000 pour avoir une vision claire du processus d'invasion du SNC par la microglie. De telles études ont pu être mises en œuvre grâce à l'utilisation d'anticorps spécifiques dirigés contre des protéines exprimées par les cellules microgliales, et surtout grâce à la production de souris exprimant la eGFP (enhanced green fluorescent protein, une protéine fluorescente) dans les cellules microgliales (souris $\mathrm{CX}_{3}$ CRleGFP) [9].

Chez la souris, les cellules microgliales envahissent la moelle épinière $(M E)$ et le cerveau au même âge embryonnaire $[10,11]$. Des macrophages immatures sont observés autour du parenchyme neural vers le $9,5^{e}-10,5^{e}$ jour après la fertilisation $(\varepsilon 9,5-\varepsilon 10,5)$, qui forment probablement un réservoir de cellules microgliales. Au niveau spinal, des macrophages immatures sortent du tractus artériel primitif dès $\varepsilon 10,5-\varepsilon 11,5$ et migrent à la périphérie de la moelle épinière [10]. Ces macrophages immatures interagissent avec les cellules endothéliales du plexus vasculaire périneural dès $\varepsilon 11,5$, et prolifèrent avant et pendant leur entrée dans le parenchyme [10]. D'abord de forme amiboïde quand elles envahissent le parenchyme, ces cellules acquièrent progressivement au cours du développement embryonnaire une morphologie ramifiée caractéristique des cellules microgliales. Les cellules amiboïdes qui s'accumulent dans la région périneurale ou qui entrent dans le parenchyme expriment d'emblée les mar- queurs décrits dans les macrophages adultes comme IBal (ionized calcium-binding adaptor molecule 1), CD68 et CD1lb [10]. À $\varepsilon 12,5$, les cellules microgliales s'accumulent transitoirement dans la région des cônes de croissance des neurones sensoriels des ganglions de la racine dorsale (nsGRD), situés au niveau dorsal latéral de la moelle épinière (Figure 2). Au niveau ventral, les cellules microgliales forment un ou deux prolongements et interagissent, d'abord avec les fibres des cellules radiaires (les précurseurs des neurones et des astrocytes) dans les faisceaux de fibres nerveuses entourant la substance grise, avant de s'accumuler transitoirement dans la région des motoneurones entre $\varepsilon 13,5$ et $\varepsilon 14,5$ (Figure 2). Chez la souris, ces agrégations ont lieu en même temps que la mort développementale (MD) par apoptose des nsGRD et des motoneurones. La $M D$ est un processus normal qui permet la sélection des neurones fonctionnels au cours du développement $d u$ SNC [10]. Après $\varepsilon 15,5$, toutes les cellules microgliales sont ramifiées et réparties de façon homogène dans le parenchyme spinal. Un processus de colonisation analogue a été observé chez l'embryon de poulet [12]. Ce processus d'invasion peut être modulé par le microenvironnement. Rigato et al. [13] ont montré, en utilisant des approches multiples et différents types de 

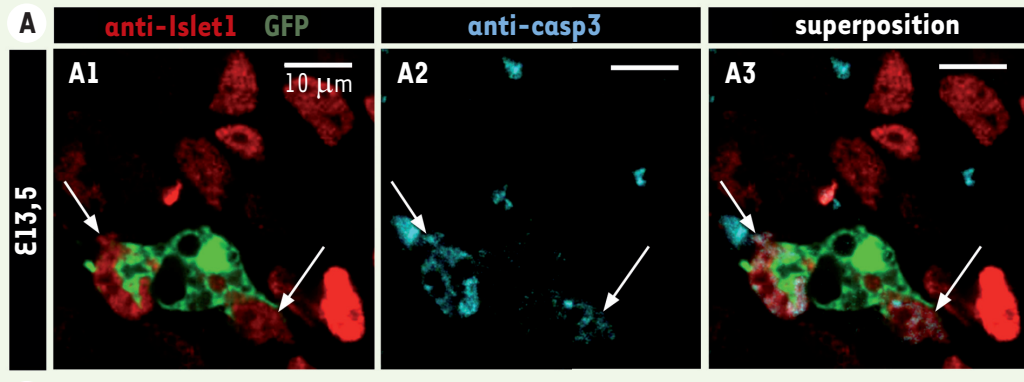

B
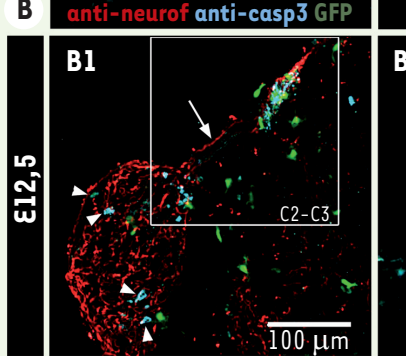

anti-casp3

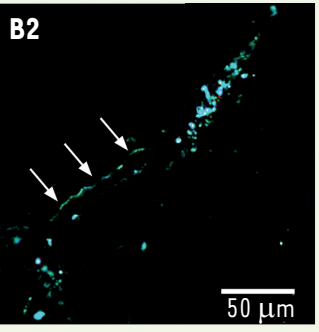

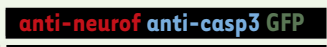

B3

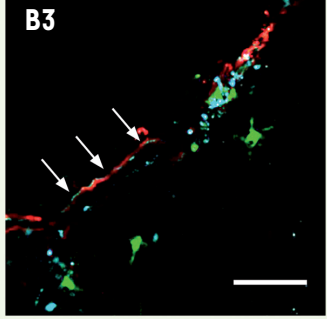

Figure 3. Les cellules microgliales embryonnaires expriment un phénotype macrophagique au début de la mort développementale des motoneurones. A. La microglie s'accumule dans les colonnes motrices au début de la mort développementale des motoneurones dans la moelle épinière embryonnaire $(\varepsilon 13,5)$. Sur la photographie $\mathbf{A l}$, on peut voir une cellule microgliale (vert) phagocytant un motoneurone. Le noyau du motoneurone (rouge) est marqué à l'aide d'un anticorps dirigé contre le facteur de transcription Isletl. L'image du milieu (A2) montre un marquage de cellule en apoptose (bleu). Dans cet exemple, l'apoptose est mise en évidence à l'aide d'un anticorps dirigé contre la caspase 3 activée. A3. Superposition des images en $\boldsymbol{A} \mathbf{1}$ et en $\boldsymbol{A} \mathbf{2}$ montrant que les motoneurones phagocytés par les cellules microgliales sont en apoptose. En $\boldsymbol{A 1}, \boldsymbol{A} 2$ et $\boldsymbol{A} \mathbf{3}$, les flèches représentent des noyaux de moto-

neurones (marquages avec un anticorps dirigé contre les facteurs de transcriptions Islet 1 et 2, en rouge) en apoptose (marquage avec un anticorps dirigé contre la caspase 3 activée : bleu) qui sont en train d'être phagocytés par une microglie (en vert). B. À El2,5, les cellules microgliales s'accumulent au voisinage des terminaisons des axones en croissance des neurones sensoriels des ganglions de la racine dorsale. Les fibres des neurones sensoriels (rouge) ont été mises en évidence en utilisant un anticorps dirigé contre des neurofilaments de 145 kDa. L'image en B2 montre un marquage immunologique avec un anticorps dirigé contre la caspase 3 activée (bleu). On remarquera que ce marquage est présent au niveau des corps cellulaires situés dans le ganglion, et aussi au niveau de la racine dorsale. B3. La superposition des images $\mathbf{B} \mathbf{1}$ et $\mathbf{B} 2$ indique que les neurones sensoriels en apoptose expriment la caspase 3 activée au niveau de leurs corps cellulaires et dans leurs axones. On voit aussi dans cette image que les cellules microgliales sont regroupées au niveau des terminaisons des neurones sensoriels en apoptose. En BI, les pointes de flèches montrent des neurones sensoriels du ganglion de la racine dorsale en apoptose (anticorps dirigé contre la caspase 3 activée en bleu). La flèche dans l'encart B1 montre une fibre allant du ganglion de la racine dorsale à la moelle épinière. En $B 2$ et en $B 3$, les flèches montrent une fibre allant du ganglion de la racine dorsale à la moelle épinière (en rouge) qui contient de la caspase 3 activée (en bleu) et qui provient d’un neurone du ganglion de la racine dorsale qui est en apoptose (marquage avec un anticorps dirigé contre la caspase 3 activée : bleu) (d'après [10]).

souris transgéniques, que la prolifération des précurseurs microgliaux dans la $M \varepsilon$ embryonnaire de souris dépendait de leur capacité à exprimer le récepteur $P 2 X_{7}\left(P 2 X_{7} R\right)$ de I'ATP [22]. Les précurseurs microgliaux ne prolifèrent plus chez des embryons de souris dépourvus de $P 2 X_{7} R$. Si leur nombre est significativement diminué, cela n'empêche pas le processus de colonisation du parenchyme spinal par la microglie. Le déficit en précurseurs microgliaux dû à la réduction de leur capacité proliférative pourrait être compensé par un apport accru de macrophages immatures qui se sont accumulés autour de la $M E$ au niveau du plexus périneuronal dès $\varepsilon 11,5$ et/ou par l'infiltration de macrophages provenant de l'hématopoï̀se définitive. Ces travaux ont, par ailleurs, montré que $P 2 X_{7} R$ n'était pas impliqué dans les processus d'activation de la microglie, contrairement à ce qui avait été observé in vitro. Le signal activant les $P 2 X_{7} R$ et sa source restent à identifier. Il est peu probable que se soit I'ATP, qui ne peut activer $P 2 X_{7} R$ qu'à des concentrations millimolaires. II pourrait en revanche s'agir de l'ADPribosylé, dont on sait qu'il est susceptible d'activer les récepteurs $\mathrm{P}_{2} \mathrm{X}_{7}$ [14]. L'ADP circulante est ribosylée par l'ADP-ribosyltransférase en présence de nicotinamide adénine dinucléotide. Ce cofacteur est libéré par les neurones [15]. Néanmoins, ce mécanisme reste à démontrer dans la $M \varepsilon$ embryonnaire.

Dans le cortex, l'invasion des cellules microgliales a lieu après $\varepsilon 10,5$ [11]. Des macrophages immatures s'accumulent d'abord à la surface piale et dans les ventricules latéraux, qui constituent ainsi deux sites potentiels d'invasion. L'invasion se développe ensuite en plusieurs phases s'étalant de $\varepsilon 11,5$ à $\varepsilon 17,5$. Une phase initiale d'invasion a lieu de $\varepsilon 10,5$ à $\varepsilon 11,5$ et est suivie d'une phase de stabilisation. À $\varepsilon 14,5$, la densité des cellules microgliales augmente brutalement dans le parenchyme, mais se stabilise après $\varepsilon 15,5$, et aucun changement n'est observé jusqu'à $\varepsilon 17,5$, le temps final de l'analyse menée dans cette étude. La phase initiale d'invasion est associée à une prolifération des macrophages immatures entourant le cortex. En revanche, il n'y a pas de relation entre leur taux de prolifération et le deuxième pic d'invasion. Comme la densité de macrophages immatures entourant le 
cortex diminue au profit de la densité des cellules microgliales dans le parenchyme, cette deuxième phase d'invasion pourrait être la conséquence de la migration des macrophages immatures provenant des zones péricorticales. De façon remarquable, des cellules microgliales s'accumulent dans les plexus choroïdes dès $\varepsilon 11,5$ au moment de la mort développementale (MD) des cellules composant les plexus choroïdes. Quand elles atteignent cette région, les cellules microgliales prolifèrent et sont capables de phagocyter les débris cellulaires [11]. À partir de $\varepsilon 17,5$, les cellules microgliales s'accumulent dans la zone sous-ventriculaire du cortex. Cette accumulation sous-ventriculaire a été observée chez la souris, le rat et le macaque $[11,16]$.

Si le changement de morphologie des cellules microgliales (d'amiboïde à ramifiée) au cours du développement à longtemps été considéré comme un index de maturation [1], des études récentes ont démontré qu'il fallait prendre ce postulat avec précaution. En effet, Swinnen et al. [11] ont montré, grâce à la vidéomicroscopie, que la microglie qui envahit le cortex embryonnaire oscille plusieurs fois en moins d'une heure entre un phénotype amiboïde et un phénotype ramifié. Bien que la signification de ces changements rapides soit encore inconnue, ils suggèrent que les cellules microgliales embryonnaires sont capables d'interagir avec leur environnement immédiat dès leur entrée dans le parenchyme.

\section{Cellules microgliales et développement du système nerveux embryonnaire : un champ de recherche en émergence}

S'il existe de plus en plus d'arguments en faveur du rôle des cellules microgliales dans la régulation du développement du SNC et de la synaptogenèse après la naissance, peu de données sont disponibles sur leur rôle éventuel au cours du développement embryonnaire.

Concernant la $M \varepsilon$ embryonnaire, les seules données qui existent se rapportent au rôle de la microglie dans la mort développementale des motoneurones. Le rôle macrophagique des cellules microgliales embryonnaires pendant la mort développementale des motoneurones et des cellules sensorielles des ganglions de la racine dorsale a été clairement démontré [10] (Figure 3). Mais, des études in vitro ont aussi suggéré que ces cellules pouvaient promouvoir la mort des motoneurones chez l'embryon. Sedel et al. ont ainsi montré, à l'aide de cultures d'explants de moelle embryonnaire de rat, que la microglie favorisait la mort développementale des motoneurones en les rendant compétents pour s'engager dans la voie apoptotique [17]. Ces auteurs ont proposé que la libération de TNF $\alpha$ par la microglie était à l'origine de cette fonction microgliale, sur la base de résultats obtenus à partir de cultures d'explants de $M E$ d'embryon de souris dont le gène codant pour le TNF $\alpha$, ou celui codant pour son récepteur (TNFR), ont été invalidés. Cependant, ces résultats n'ont pu être reproduits in vivo. Les analyses menées sur des embryons entiers de souris mutantes, dont les gènes codant pour le TNF $\alpha$ et son récepteur TNFR ont été invalidés, n'ont pas révélé de différence dans le nombre de motoneurones entre les mutants et les animaux non mutés au stade adulte. Ces données contradictoires, obtenues in vitro et in vivo, pourraient s'expliquer par des mécanismes de compensations chez les souris mutantes [17], mais en l'absence de données complémentaires, le rôle de la microglie dans la régulation de la mort cellulaire développementale des motoneurones reste hypothétique.

La première démonstration convaincante d'un rôle de la microglie dans la neurogenèse n'a été faite que récemment [18]. En combinant des marquages immunohistochimiques chez le macaque, le rat et la souris, et la technique d'ablation pharmacologique des cellules microgliales in vitro et in vivo, Cunningham et al. ont montré que les cellules microgliales régulent le nombre des cellules progénitrices neurales dans le cortex en développement [18]. À la fin de la neurogenèse corticale, les cellules microgliales phagocytent des précurseurs neuraux situés dans la zone ventriculaire et sous-ventriculaire, et ce en l'absence de tout signe détectable de mort cellulaire. Cette étude montre que l'activation des cellules microgliales embryonnaires par le lipopolysaccharide, un composant essentiel de la membrane externe des bactéries à Gram négatif, augmente la phagocytose des précurseurs neuraux et en fait diminuer leur nombre. Bien que le rôle de l'activation microgliale dans des pathologies neuro-développementales fait encore largement débat, les travaux de Cunningham et al. offrent une piste de recherche pour explorer les conséquences de l'altération du phénotype microglial pendant la gestation sur le développement du SNC [18].

Chez l'embryon, la microglie n'agirait pas seulement sur le développement neuronal, mais aussi sur le développement de la vascularisation du SNC (voir pour revue [19]). Dans le SNC, le développement des vaisseaux sanguins se fait exclusivement par angiogenèse. Au cours du développement embryonnaire, les vaisseaux en croissance font de façon itérative des extensions, des branchements et de l'anastomose vers le centre du tube neural où ils forment un plexus transitoire autour des ventricules du cerveau et autour du canal central de la ME [19]. C'est durant la formation de ces plexus vasculaires transitoires que les cellules microgliales contactent les vaisseaux sanguins. Le rôle de la microglie dans l'angiogenèse a été mis en évidence grâce à l'utilisation de souris dépourvues de cellules microgliales (les souris transgéniques PU.1KO et les souris mutantes ostéopétrotique csfl [colony stimulating factor 1] op/op) et dans des modèles d'ablation pharmacologique de la microglie [19]. Les souris transgéniques PU.1KO meurent à la naissance et sont aussi dépourvues de macrophages, de lymphocytes $B$, de mastocytes, de neutrophiles et d'érythroblastes précoces, 
alors que les souris csfl op/op sont dépourvues de macrophages et d'ostéoclastes. Dans ces modèles, la suppression de la microglie au cours du développement aboutit à une diminution des branchements vasculaires dans la rétine et le cerveau [19]. Cependant, les mécanismes moléculaires qui sous-tendent le rôle de la microglie dans la mise en forme du plexus vasculaire naissant sont encore inconnus [19].

L'ensemble de ces découvertes ouvrent un nouveau champ de recherche qui permettra de mieux comprendre comment les interactions entre la microglie et les neurones régulent le développement du SNC et pourraient être à l'origine de pathologies neuro-développementales. $\diamond$

\section{SUMMARY}

Microglial cells and development of the embryonic central nervous system

Microglia cells are the macrophages of the central nervous system with a crucial function in the homeostasis of the adult brain. However, recent studies showed that microglial cells may also have important functions during early embryonic central nervous system development. In this review we summarize recent works on the extra embryonic origin of microglia, their progenitor niche, the pattern of their invasion of the embryonic central nervous system and on interactions between embryonic microglia and their local environment during invasion. We describe microglial functions during development of embryonic neuronal networks, including their roles in neurogenesis, in angiogenesis and developmental cell death. These recent discoveries open a new field of research on the functions of neural-microglial interactions during the development of the embryonic central nervous system. $\diamond$

\section{LIENS D'INTÉRÊT}

Les auteurs déclarent n'avoir aucun lien d'intérêt concernant les données publiées dans cet article.

\section{RéFÉRENCES}

1. Kettenmann H, Hanisch UK, Noda M, Verkhratsky A. Physiology of microglia. Phys Rev 2011 ; 91 : 461-553.

2. Pont-Lezica L, Bechade C, Belarif-Cantaut $Y$, et al. Physiological roles of microglia during development. J Neurochem $2011 ; 119$ : 901-8.

3. Roumier A, Pascual 0 , Bechade $C$, et al. Prenatal activation of microglia induces delayed impairment of glutamatergic synaptic function. PLoS One 2008; 3 : e2595.

4. McKercher SR, Torbett BE, Anderson KL, et al. Targeted disruption of the PU.l gene results in multiple hematopoietic abnormalities. EMBO J 1996 ; 15 : 5647-58.
5. Alliot F, Godin I, Pessac B. Microglia derive from progenitors, originating from the yolk sac, and which proliferate in the brain. Brain Res Dev Brain Res $1999 ; 117: 145-52$.

6. Ginhoux F, Merad M. Les cellules de la microglie : leurs origines extraembryonnaires enfin révélées. Med Sci (Paris) 2011 ; 27 : 719-24.

7. Ginhoux F, Greter M, Leboeuf M, et al. Fate mapping analysis reveals that adult microglia derive from primitive macrophages. Science $2010 ; 330$ : 841-5.

8. Kierdorf K, Erny D, Goldmann T, et al. Microglia emerge from erythromyeloid precursors via Pu.1- and Irf8-dependent pathways. Nat Neurosci 2013; 16 : 273-80.

9. Wolf $\mathrm{Y}$, Yona S, Kim KW, Jung S. Microglia, seen from the CX3CRl angle. Front Cell Neurosci $2013 ; 7: 26$.

10. Rigato C, Buckinx R, Le Corronc H, et al. Pattern of invasion of the embryonic mouse spinal cord by microglial cells at the time of the onset of functional neuronal networks. Glia 2011 ; 59 : 675-95.

11. Swinnen N, Smolders S, Avila A, et al. Complex invasion pattern of the cerebral cortex bymicroglial cells during development of the mouse embryo. Glia $2013 ; 61: 150-63$.

12. Caldero J, Brunet N, Ciutat D, et al. Development of microglia in the chick embryo spinal cord: implications in the regulation of motoneuronal survival and death.J Neurosci Res $2009 ; 87$ : 2447-66.

13. Rigato C, Swinnen N, Buckinx R, et al. Microglia proliferation is controlled by P2X7 receptors in a Pannexin-1-independent manner during early embryonic spinal cord invasion. J Neurosci $2012 ; 32$ : 11559-73.

14. Hong S, Schwarz N, Brass A, et al. Differential regulation of $P 2 X 7$ receptor activation by extracellular nicotinamide adenine dinucleotide and ectoADP-ribosyltransferases in murine macrophages and T cells. J Immunol $2009 ; 183: 578-92$.

15. Durnin L, Dai Y, Aiba I, et al. Release, neuronal effects and removal of extracellular beta-nicotinamide adenine dinucleotide (beta-NAD+) in the rat brain. EurJ Neurosci 2012 ; 35 : 423-35.

16. Ueno $M$, Fujita $Y$, Tanaka $T$, et al. Layer $V$ cortical neurons require microglial support for survival during postnatal development. Nat Neurosci $2013 ; 16$ : 543-51.

17. Sedel F, Bechade C, Vyas S, Triller A. Macrophage-derived tumor necrosis factor alpha, an early developmental signal for motoneuron death. J Neurosci $2004 ; 24: 2236-46$.

18. Cunningham CL, Martinez-Cerdeno V, Noctor SC. Microglia regulate the number of neural precursor cells in the developing cerebral cortex. J Neurosci $2013 ; 33: 4216-33$.

19. Arnold T, Betsholtz C. The importance of microglia in the development of the vasculature in the central nervous system. Vasc Cell $2013 ; 5: 4$.

20. Neumann $\mathrm{H}$, Wekerle $\mathrm{H}$. Brain microglia: watchdogs with pedigree. Nat Neurosci $2013 ; 16: 253-5$.

21. Audinat $\varepsilon$, Arnoux I. La microglie : des cellules immunitaires qui sculptent et contrôlent les synapses neuronales. Med Sci (Paris) 2014 ; $30: 153-9$.

22. Pochet $S$, Seil M, El Ouaaliti M, Dehaye JP. P2 $X_{4}$ ou P2X $X_{7}$ : lequel de ces deux récepteurs nous fera saliver? Med Sci (Paris) 2013 ; 29 : 509-14.

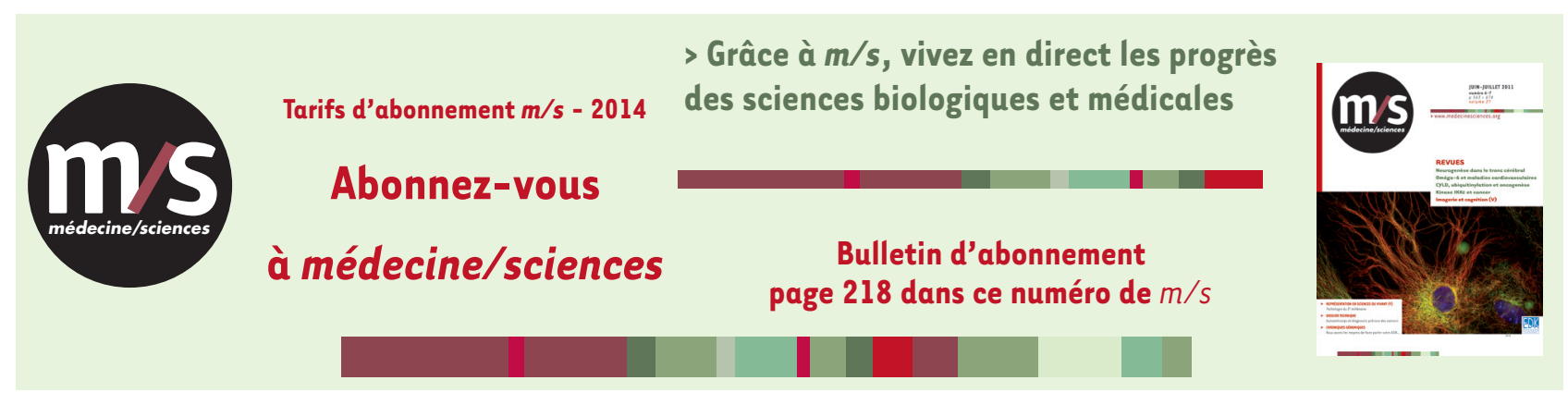

TIRÉS À PART

P. Legendre 